\title{
Simulation and Analysis of the Radiation Pattern of Microstrip Patch-Type Array Antenna by Moment Method
}

\author{
Sergio Martínez Campo ${ }^{1}$, Mario Orlando Oliveira ${ }^{2}$, and Libis Valdez Cervantes ${ }^{1}$ \\ ${ }^{1}$ Fundación Tecnológica Antonio de Arévalo, Colombia, sergio.martinez@tecnar.edu.co, libis.valdez@tecnar.edu.co \\ ${ }^{2}$ Universidad Nacional de Misiones, Argentina, oliveira@fio.unam.edu.ar
}

\begin{abstract}
This paper presents the results of a research project aimed at studying the behavior of the radiation pattern of a patch array antenna through computer simulations. In this sense, we present the basic guidelines for design, implementation and preliminary simulation of different antenna prototypes through an algorithm simulator developed in MATLAB ${ }^{\circledR}$ environment. Comparative studies of the simulation values were performed with the software LVDAM-ANT ${ }^{\circledR}$ which relate simulation data with actual experimental values. The results obtained demonstrate the efficiency of the simulation algorithm developed to underpin the study presented in this paper.

Keywords- Microstrip patch array, radiation pattern, electromagnetic field, simulator GUI.
\end{abstract}

Digital Object Identifier (DOI): http://dx.doi.org/10.18687/LACCEI2015.1.1.279

ISBN: 13 978-0-9822896-8-6

ISSN: $2414-6668$

$1^{\text {th }}$ LACCEI Annual International Conference: “Engineering Education Facing the Grand Challenges, What Are We Doing?” July 29-31, 2015, Santo Domingo, Dominican Republic ISBN: 13 978-0-9822896-8-6

ISSN: 2414-6668

DOI: http://dx.doi.org/10.18687/LACCEI2015.1.1.279 


\title{
Simulation and Analysis of the Radiation Pattern of Microstrip Patch-Type Array Antenna by Moment Method
}

\author{
Sergio Martínez Campo ${ }^{1}$, Mario Orlando Oliveira ${ }^{2}$, and Libis Valdez Cervantes ${ }^{1}$ \\ ${ }^{1}$ Fundación Tecnológica Antonio de Arévalo, Colombia, sergio.martinez@tecnar.edu.co, libis.valdez@tecnar.edu.co \\ ${ }^{2}$ Universidad Nacional de Misiones, Argentina, oliveira@fio.unam.edu.ar
}

\begin{abstract}
This paper presents the results of a research project aimed at studying the behavior of the radiation pattern of a patch array antenna through computer simulations. In this sense, we present the basic guidelines for design, implementation and preliminary simulation of different antenna prototypes through an algorithm simulator developed in MATLAB ${ }^{\circledR}$ environment. Comparative studies of the simulation values were performed with the software $L V D A M-A N T{ }^{\circledR}$ which relate simulation data with actual experimental values. The results obtained demonstrate the efficiency of the simulation algorithm developed to underpin the study presented in this paper.
\end{abstract}

Keywords- Microstrip patch array, radiation pattern, electromagnetic field, simulator GUI.

\section{INTRODUCCIÓN}

Las antenas son el componente fundamental de los sistemas de telecomunicación, las cuales han alcanzado un elevado desarrollo tecnológico (alto rendimiento y miniaturización de componentes). Las aplicaciones se han expandido con los años y el fin de su miniaturización ha hecho de éstas una importante e interesante base para la demanda en la comercialización y posterior implementación con distintos instrumentos electrónicos. En este sentido, el tamaño, el peso, el costo, la facilidad de instalación y el contorno aerodinámico son parámetros de suma importancia. Los diversos tipos de antenas están relacionados con las características del campo electromagnético y con la tecnología utilizada, según el uso al que vaya ser destinada. En 1981 [1], se presenta un estudio del comportamiento de las antenas reactivamente cargadas, el cual, se dio solución con el método de los momentos para una antena microstrip de parche rectangular. El objetivo principal ha sido diseñar la antena ofreciendo diversidad de polarización y de frecuencia. El modo de cinta dual es caracterizado por una ranura delgada además de líneas microstrip como una antena cargada. En [2] se presenta una antena microstrip tipo parche de banda ancha para comunicación inalámbrica. En 2008, en [3] se presenta un modelo convencional de línea de transmisión para estudio de antenas microstrip, pero no propone una herramienta computacional para obtener los campos electromagnéticos ni el diagrama de radiación. El mismo año, [4] y [5] utilizan una herramienta computacional para simular los diagramas de radiación de una antena microstrip, sin embargo, solo analizan una antena parche rectangular. En 2009, [6] utiliza una herramienta de simulación que analiza solo la antena rectangular microstrip, no simula un arreglo de antenas rectangulares. En [7] y [8] se realiza un estudio utilizando los datos de simulación del diagrama de radiación de una antena específica pero no realizan la comparación de los resultados con datos experimentales. En 2013, [9] presenta un procedimiento para el diseño de una antena parche utilizando el modelo de línea de transmisión. Este analiza un ejemplar de antena microstrip, el cual determina que el tamaño de la antena microstrip es inversamente proporcional a su frecuencia de funcionamiento, pero no propone una herramienta computacional para obtener los campos electromagnéticos ni el diagrama de radiación y su respectivo análisis. En [10] utiliza un simulador basado en el método de los momentos donde diseña y analiza antenas microstrip parche rectangular, sin embargo no diseñan ni simulan un arreglo de parche rectangulares y no comparan los resultados con datos experimentales. También en 2013, [11] presenta un análisis del diagrama de radiación de una antena microstrip rectangular por medio del modelo de línea de transmisión para el diseño y modelo de concavidad para la simulación, donde se desarrolla una herramienta de simulación computacional para obtener los diferentes diagramas de radiación. Mediante un análisis estadístico y comparación de los resultados simulados con datos obtenidos por el módulo LVDAM-ANT, muestran la excelente eficiencia del simulador.

El parche puede tomar cualquier forma posible. Teniendo en cuenta la gran versatilidad y variedad de las antenas, el presente artículo muestra un análisis del diagrama de radiación de una antena microstrip con arreglos de parche rectangular, donde se desarrolla una herramienta de simulación computacional basada en el método de los momentos para obtener los diagramas de radiación. Adicionalmente se comparan los resultados de simulación con datos experimentales obtenidos por el módulo LVDAM-ANT.

\section{TECNOLOGÍA MiCROSTRIP Y MÉTODOS DE ANÁLISIS}

Es una tecnología planar que permite guiar y radiar ondas electromagnéticas mediante estructuras impresas (denominada comúnmente parche) en un substrato dieléctrico totalmente metalizado por una de sus caras como se muestra en la Fig. 1. Es la tecnología de elección en la mayor parte de los dispositivos móviles de telecomunicación actuales. La Fig. 1 muestra la forma básica y las medidas más importantes de una antena tipo microstrip.

Existen varios métodos de análisis para diseño de antenas tipo parche o microstrip. Dependiendo de la precisión y el grado de sencillez que se busque se puede seleccionar el método que más se ajuste a las necesidades [12]. En este trabajo se utilizó el método de los momentos para el análisis del diagrama de radiación de la antena. 


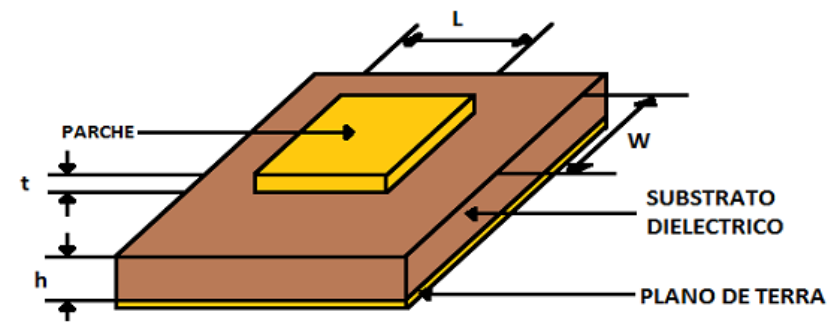

Figura 1. Forma y dimensiones de la antena microstrip.

Consiste de un parche conductor con dimensiones de largo $L$, ancho $W$ y grosor $t$, separadas de un plano de tierra por una lámina delgada de material dieléctrico con ancho $h$ [12].

\section{A. Factor de Arreglo}

El cálculo de las dimensiones de la antena microstrip usando los parámetros utilizados en [11] se muestra en (1) y (2).

$$
l=\frac{c}{2 f_{0} \sqrt{\varepsilon_{r}}}
$$

donde

$$
\begin{gathered}
f_{0}=10 \cdot 10^{9} \\
\varepsilon_{r}=4
\end{gathered}
$$

(1a) es la frecuencia de trabajo para la antena microstrip y (1b) es la permitividad del dieléctrico.

$$
w=\frac{c}{2 f_{0} \sqrt{\frac{\varepsilon_{r}+1}{2}}}
$$

El Factor de Arreglo empleado en la construcción del arreglo lineal de parches rectangulares se representa en la siguiente ecuación.

$$
F A=\frac{1}{n} \cdot \frac{\operatorname{Sin}\left[\frac{n}{2} \cdot y_{x}\right]}{\operatorname{Sin}\left[\frac{y_{x}}{2}\right]}
$$

donde

$$
\begin{aligned}
& y_{x}=\left[k \cdot d_{x} \cdot \operatorname{Sin}[\theta] \cdot \operatorname{Sin}[\phi]\right]+b_{x} \\
& b_{x}=\left[-k \cdot d_{x} \cdot \operatorname{Sin}\left[\theta_{0}\right] \cdot \operatorname{Sin}\left[\phi_{0}\right]\right]
\end{aligned}
$$

$n$ es el número de parches en el eje $x$.

Los rangos de medidas del parche deben cumplir con ciertas características tales como [12]:

- $t$ debe ser delgado: $t<<\lambda_{0}\left(\lambda_{0}\right.$ : longitud de onda de la señal en el espacio libre) situada sobre el plano de tierra con un $h \ll \lambda_{0}\left(0,003 \lambda_{0} \leq h \leq 0,05 \lambda_{0}\right)$.

- $L$ puede variar dependiendo de la dimensión estipulada. Para un parche tipo rectangular se tienen los siguientes rangos: $\lambda_{0} / 3<L<\lambda_{0} / 2$.
- el ancho del substrato debe de ser mucho menor que la longitud de onda: $h<<\lambda$.

- la permitividad eléctrica del substrato queda entre: $2,2 \leq$ $\varepsilon r \leq 12$. Siempre debe buscarse la menor permitividad posible para lograr una mejor eficiencia de la antena.

\section{B. Tipos de Ondas}

Existen 4 tipos de ondas presentes en una línea de microstrip: ondas espaciales (space waves), ondas superficiales (surface waves), ondas de fuga (leaky waves) y ondas guía (guide waves) [12].

1) Ondas Espaciales: Son aquellas ondas radiadas por fuera de la estructura. Son enviadas al espacio libre, pierden magnitud y se atenúan conforme aumenta la distancia.

2) Ondas Superficiales: Son aquellas que se presentan en dirección descendente y limitadas. Están confinadas prácticamente dentro del dieléctrico (parche) y la placa metálica (plano de tierra). Es caracterizada por su uniformidad y disminución exponencial.

3) Ondas de Fuga: Son parecidas a las ondas de superficie, con diferencia en que cuando son reflejada de vuelta del plano tierra, una parte de las ondas se refleja nuevamente mientras que la otra se fuga al espacio libre. Las ondas de fuga pueden ser utilizadas en algunas estructuras como parches apilados para aumentar las dimensiones aparentes de las antenas y poder tener una ganancia más amplia.

4) Ondas Guiadas: Se presentan en circuitos impresos que son utilizados para guías de onda o líneas de transmisión. Las ondas guiadas se encuentran viajando dentro del substrato rebotando tanto en la parte metálica superior como en la que está referenciada a tierra. Esta estructura no es utilizada para aplicaciones de antenas.

Cada modo lleva asociado un diagrama de radiación diferente. Es importante resaltar que el modelo de cavidad, igual que el de línea de transmisión, no tiene en cuenta la radiación producida en la antena dado que una cavidad por definición no deja escapar energía. Por medio del método de los momentos, se puede analizar los acoples entre antenas (de aplicación en arrays), caracterizar de forma rigurosa la impedancia de entrada de antenas con configuraciones de alimentación complejas, etc. [12].

\section{Método de los Momentos}

Las corrientes superficiales se utilizan para modelar el parche microstrip y el volumen de polarización de las corrientes por el bloque dieléctrico. Hallar la distribución de la carga en la superficie de un sistema de conductores, conocido el potencial a que se encuentra cada uno de ellos. A partir de la distribución de carga se obtiene, de forma directa, el campo y el potencial en cualquier punto del espacio [12].

La Fig. 2 ilustra el uso del método en electromagnetismo, se aplicará el cálculo de la distribución de carga sobre la superficie de un conductor a potencial conocido $V o$. 


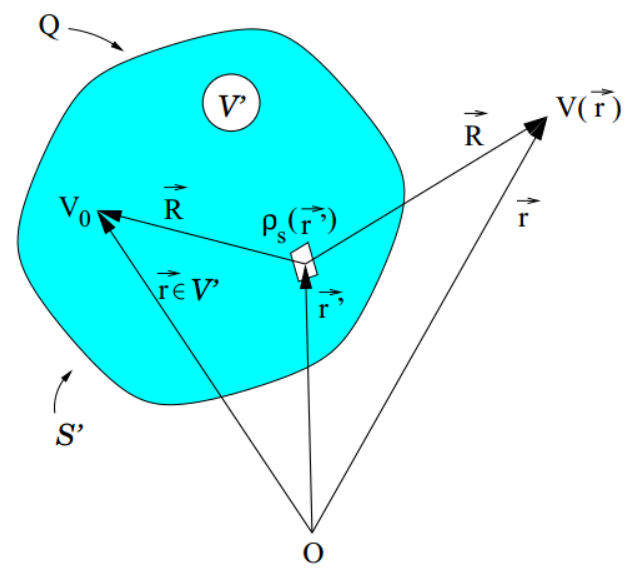

Figura 2. Distribución de Carga [12].

La Ec. (4) (ecuación de Poisson) es la expresión integral de la distribución de carga, siendo tanto $V(\vec{r})$ como $\rho(\vec{r})$ desconocidos, salvo en el conductor, puesto que está al potencial Vo. La solución puede abordarse numéricamente a partir de su expresión integral [12].

$$
V(\vec{r})=\frac{1}{4 \pi \varepsilon_{0}} \int_{S^{\prime}} \frac{\rho_{S}\left(\vec{r}^{\prime}\right) d s^{\prime}}{R}
$$

tomando a $\vec{r}$ en el interior del conductor

$$
V_{0}=\frac{1}{4 \pi \varepsilon_{0}} \int_{S^{\prime}} \frac{\rho_{S}\left(\overrightarrow{r^{\prime}}\right) d s^{\prime}}{R}
$$

donde $V o$ es el potencial en cualquier punto del interior del volumen $V^{\prime}$ del conductor, $R$ la distancia entre el punto interior $\vec{r} \in V$, en el que se calcula el potencial, y otro genérico de $\vec{r} \in S^{\prime}$, en el que se evalúa la densidad. Como se representa en la Fig. 2, el potencial se calcula en un punto de $S^{\prime}$, en su proximidad $\left(\vec{r} \in S^{\prime} \rightarrow \vec{r}^{\prime}\right.$ ) y $\frac{1}{R} \rightarrow \infty$, por lo que la integral presenta una singularidad en $\vec{r} \equiv \vec{r}^{\prime}$. Esta dificultad puede soslayarse colocando los puntos de observación en el interior de $V$ [12].

La solución de la Ecu. (6) conduce a la obtención de la densidad superficial de carga sobre el conductor.

$$
£\left(\overrightarrow{\mathrm{r}}, \overrightarrow{\mathrm{r}}^{\prime}\right)\left[\mathrm{f}\left(\overrightarrow{\mathrm{r}}^{\prime}\right)\right]=g(\overrightarrow{\mathrm{r}})
$$

donde $£$ es un operador integral lineal que, aplicado a una función $\mathrm{f}=\mathrm{f}\left(\overrightarrow{\mathrm{r}}^{\prime}\right)$ desconocida, da como resultado una función $g(\overrightarrow{\mathrm{r}})$ conocida.

$$
£[]=\frac{1}{4 \pi \varepsilon_{0}} \int_{S^{\prime}},[] \frac{d s^{\prime}}{R}, \mathrm{f}\left(\overrightarrow{\mathrm{r}}^{\prime}\right)=\rho\left(\overrightarrow{\mathrm{r}}^{\prime}\right), g(\overrightarrow{\mathrm{r}})=V_{0}
$$

Para resolver la Ecu. (6), elegimos una base $\left\{\mathrm{f}_{\mathrm{j}}\left(\overrightarrow{\mathrm{r}}^{\prime}\right)\right\}$ de dimensión $N$, para aproximar la solución de la forma

$$
\mathrm{f}\left(\overrightarrow{\mathrm{r}}^{\prime}\right) \cong \overline{\mathrm{f}}\left(\overrightarrow{\mathrm{r}}^{\prime}\right)=\alpha_{j} \mathrm{f}_{\mathrm{j}}\left(\overrightarrow{\mathrm{r}}^{\prime}\right)
$$

donde $\left\{\alpha_{\mathrm{j}}\right\}$ serán también los coeficientes a determinar.

Dada la linealidad del operador $£$, la introducción de la expresión (7) en la ecuación (6), da lugar a una versión aproximada.

$$
\alpha_{\mathrm{j}} \mathrm{£f}_{\mathrm{j}}\left(\overrightarrow{\mathrm{r}}^{\prime}\right)=g(\overrightarrow{\mathrm{r}})
$$

A continuación se eligen $N$ funciones de peso $\left\{p_{i}(\vec{r})\right\} \mathrm{y}$ se halla un producto interno de la ecuación (8) con los mismos.

$$
\alpha_{\mathrm{j}}\left\langle\mathrm{p}_{\mathrm{i}}, \mathrm{Ef}_{\mathrm{j}}\right\rangle=\left\langle\mathrm{p}_{\mathrm{i}}, \mathrm{g}\right\rangle
$$

Este sistema de ecuaciones, en el que los productos internos son escalares conocidos y las $\alpha_{\mathrm{j}}$ son las incógnitas, puede escribirse en la forma matricial.

$$
A_{i j} x_{j}=\mathrm{b}_{i}
$$

donde

$$
A_{i j}=\left\langle\mathrm{p}_{\mathrm{i}}, £ \mathrm{f}_{\mathrm{j}}\right\rangle, b_{i}=\left\langle\mathrm{p}_{\mathrm{i}}, \mathrm{g}\right\rangle, x_{j}=\alpha_{\mathrm{j}}
$$

La solución es:

$$
f \cong \alpha_{j} f_{j}
$$

Para determinar la distribución de corriente en una antena lineal, resultado de una excitación arbitraria puede ser establecido en términos de una ecuación integral. Esta ecuación emplea una función el cual relaciona un campo eléctrico conocido de las condiciones de contorno con una distribución desconocida de corriente en la antena. El método de los momentos (MoM) aplica expansiones para convertir la ecuación integral en un sistema de ecuaciones lineales. Funciones de base son usadas para la expansión de la corriente y funciones de prueba para el campo eléctrico. La distribución de corrientes es construida de los coeficientes de la expansión. Las características de la radiación de la antena son derivadas luego del cálculo de la distribución de corriente [12].

\section{SIMULADOR GUI PROPUESTO}

GUI es un algoritmo de simulación creado en ambiente Matlab® con entorno visual cuyo objetivo es permitir el diseño y análisis de arreglo (array) lineal de parche rectangular de antenas microstrip. El simulador está desarrollado con un algoritmo el cual realiza todos los cálculos y gráficas para poder representarlas en la interface.

Los arreglos de antenas se construyen con repeticiones de elementos radiantes formando un conjunto lineal. La forma de estos parches puede ser idéntica o diferente. Todos los elementos tienen una distancia, el cual puede ser la misma o variar dependiendo de la configuración de cada parche rectangular.

\section{A. Interface Gráfica}

En primer paso se deben ingresar los valores del material y la frecuencia de operación $\left(\varepsilon_{r}, h, f_{r}\right)$ en la sección "Entradas", mostrado en la Fig. 3 


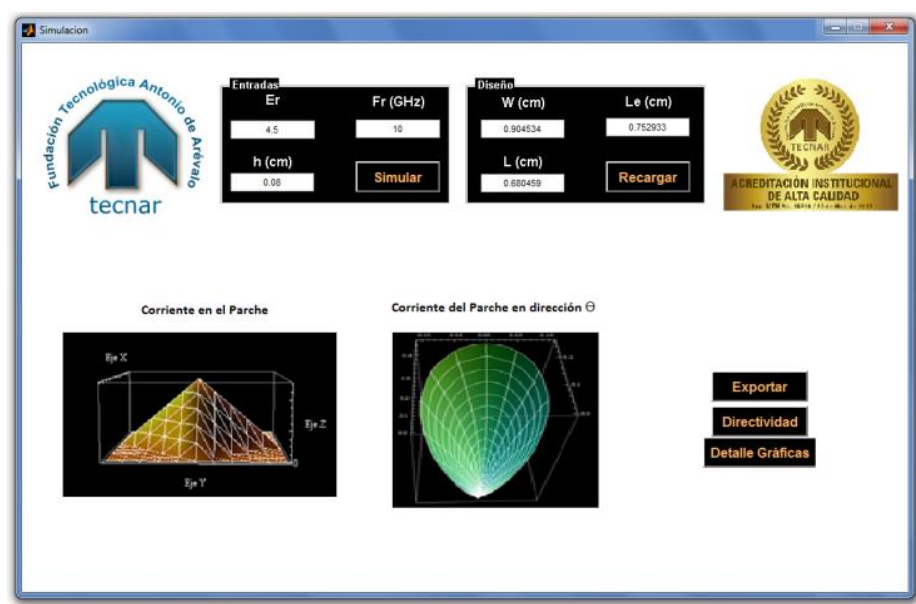

Figura 3. Menú principal del GUI con resultados de la simulación

Posteriormente, se ingresa las dimensiones del parche unificado para el arreglo, ( $W$ y $L$ ) de la antena.

También es posible obtener gráficas detalladas a través de la opción "Detalle Gráficas" localizado en el menú principal. Las simulaciones realizadas pueden ser guardadas con extensión .DAT, .TXT y .XLSX, en forma matricial y vectorial, en una ruta seleccionada por el usuario con la opción "Exportar". De modo que se pueda comparar los datos simulados con los datos experimentales obtenidos a través del módulo Lab-Volt ${ }^{\circledR}$ - Antenna Training and Measuring System (8092) y el software LVDAM-ANT [13].

Tiene opción de graficar los diagramas de radiación en Polar y de igual forma exportarlos. Además calcula y gráfica la impedancia de entrada dependiendo de la configuración de cada parche rectangular.

\section{ESTUDIO DE CASO}

Esta sección presenta un estudio de caso realizado con el fin de verificar la eficiencia del simulador propuesto. Para tal fin se pretende proyectar un arreglo lineal de antena microstrip con frecuencia de operación de $10 \mathrm{Ghz}$ construida con un material dieléctrico de FR4 1/1 (fibra de vidrio). La constante dieléctrica es $\varepsilon_{r}=4.5$ y tiene un espesor $h=0.8 \mathrm{~mm}$. Estos datos son cargados en el menú principal del simulador GUI como datos de entrada y el mismo retorna las dimensiones $W$ y $L$. Este procedimiento es similar al utilizado en [11].

Se simuló y construyó un arreglo de parche, con las siguientes medidas: $W=1,2 \mathrm{~cm}, L=0,7 \mathrm{~cm}$.

\section{A. Ensayo Experimental}

Con el prototipo construido según los valores simulados se realizó la toma de datos de laboratorio a través del módulo Lab-Volt@ - Antenna Training and Measuring System (8092) y el software LVDAM-ANT [13]. La Fig. 4 muestra el prototipo de un arreglo de parche lineal antena microstrip.

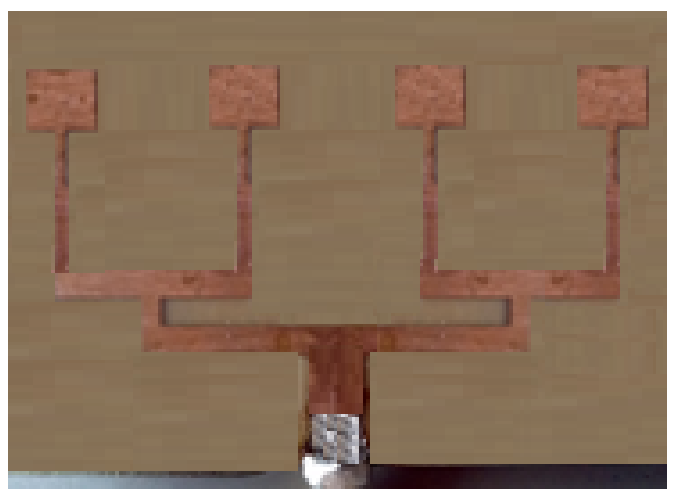

Figura 4. Antena Microstrip (4x1)

En la Fig. 5 se puede observar los diagramas de radiación del campo eléctrico (rojo) y campo magnético (azul) medido por el LVDAM-ANT.

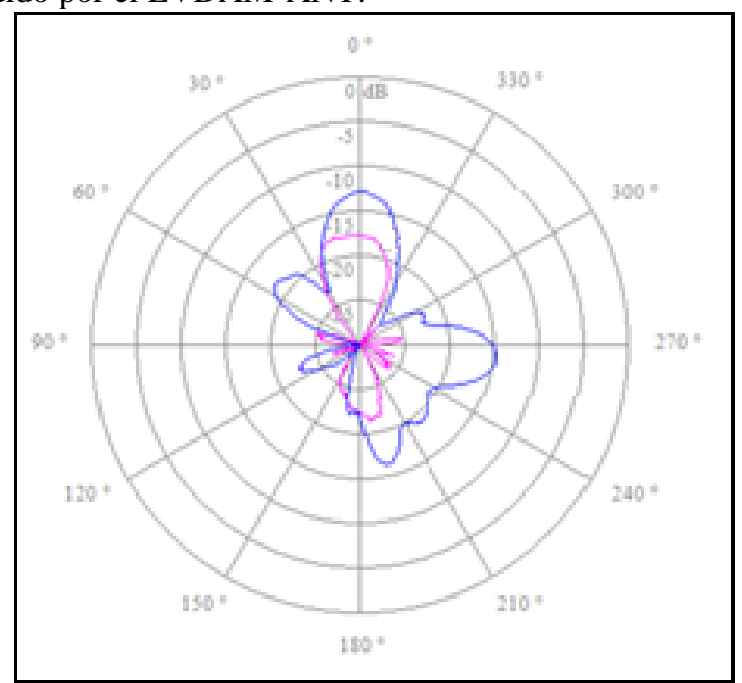

Figura 5. Diagramas de Radiación obtenidos por el módulo LVDAM-ANT

El lóbulo trasero que presenta el campo eléctrico se debe al ruido generado por el cable de alimentación, que trata de radiar e interfiere en el diagrama de radiación.

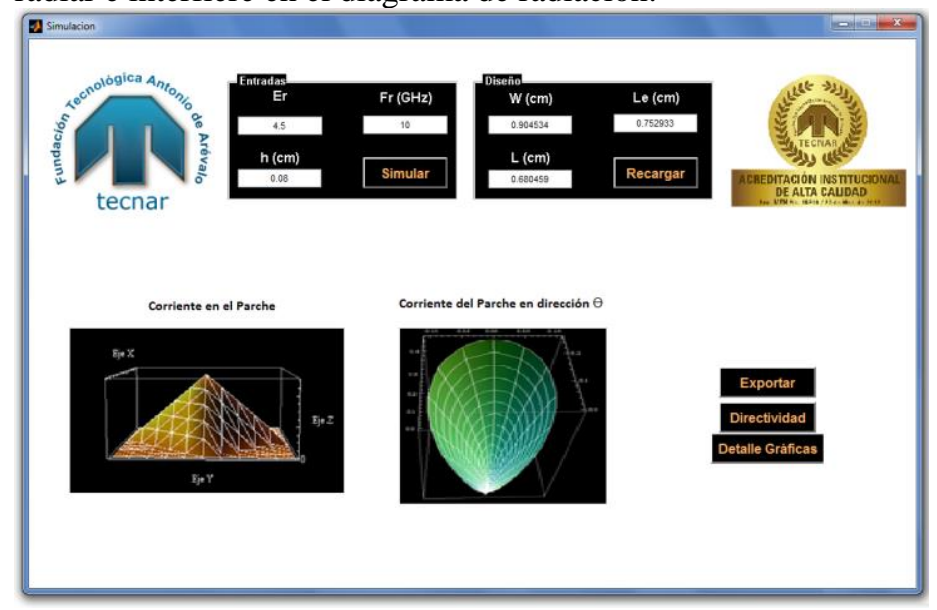

Figura 6. Simulación del estudio de caso

La Fig. 6 muestra la representación de los diagramas de radiación y la corriente del parche, el cual, muestra todas las opciones para exportar y analizar la simulación. 


\section{ANÁLISIS DE RESULTADOS}

La Fig. 7 muestra la variación de la impedancia de entrada en función del campo eléctrico y campo magnético a la frecuencia específica del diseño.

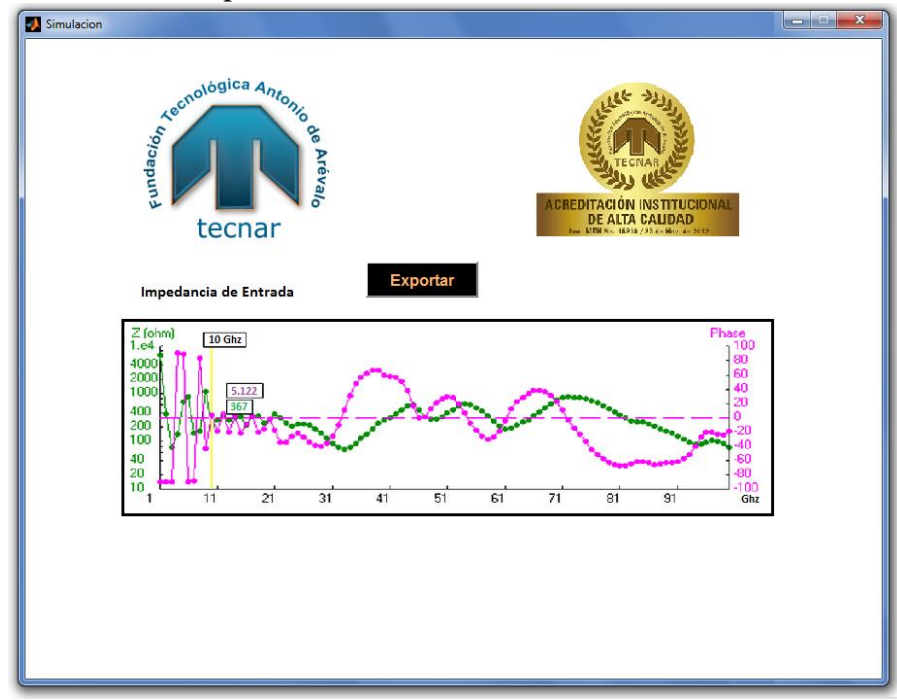

Figura 7. Impedancia de Entrada del Estudio de Caso

Con el análisis de los diagramas de radiación, de la variación de los materiales de construcción de la antena, y las diferentes dimensiones del parche rectangular, realizado en [11], se pudo seleccionar los materiales y las dimensiones para el arreglo lineal según nuestras necesidades del proyecto en el caso estudio.

La Fig. 8 muestra la representación de los lóbulos de los diagramas de radiación en Polar, además de la magnitud normalizada versus ángulos. En la Fig. 9, se puede observar la gráfica de los datos experimentales importados en el Simulador GUI, con el fin de realizar comparaciones gráficas y estadísticas.

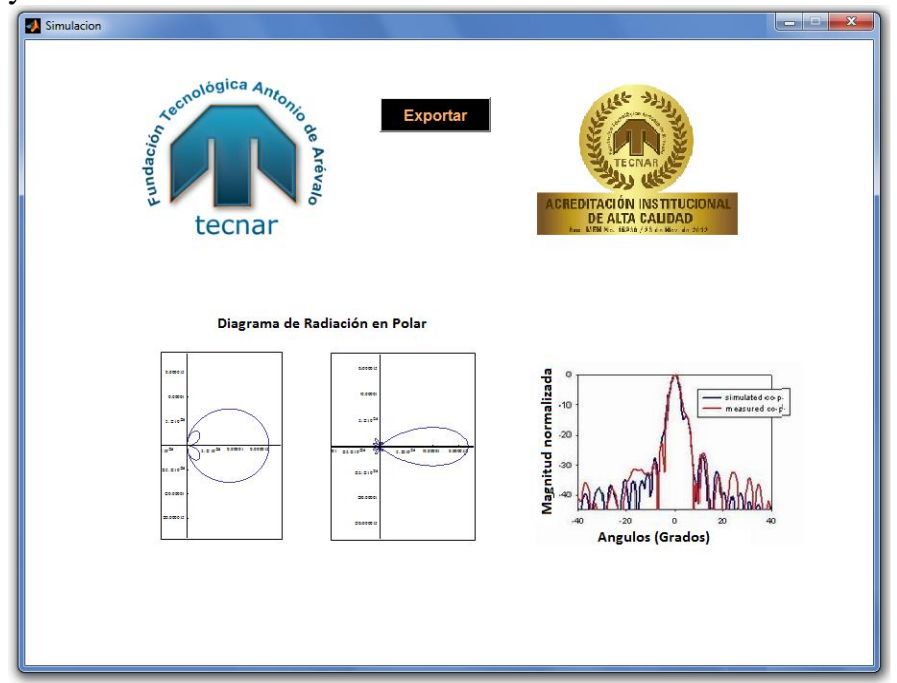

Figura 8. Representación Gráfica en Polar y Magnitud Normalizada Vs Ángulos

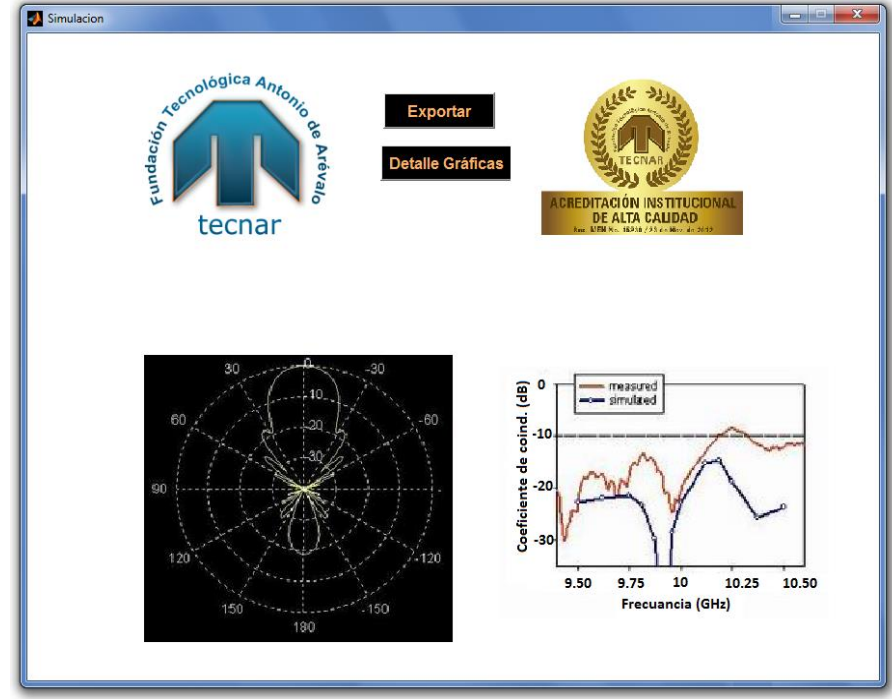

Figura 9. Gráfica de los valores experimentales obtenidos en el Módulo LVDAM-ANT e importador al simulador.

El simulador GUI, tiene la opción determinar y analizar la directividad de la antena. La Fig. 10 muestra la representación de la directividad en función de $L$ de la antena de caso estudio.

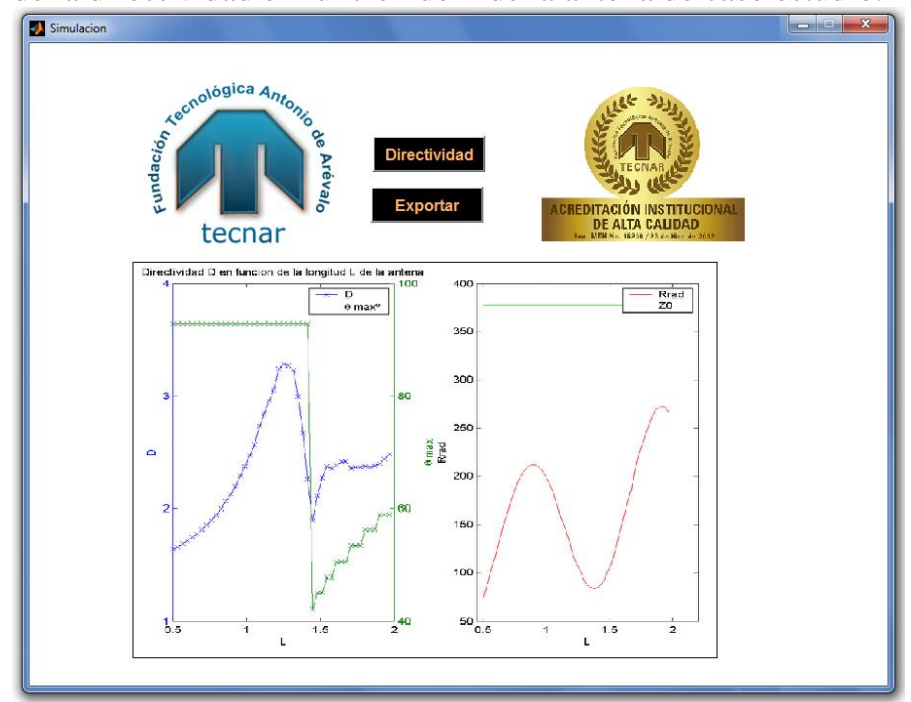

Figura 10. Directividad en función de la dimensión $L$ del parche.

A fines de validar los datos obtenidos por el simulador GUI, se realizó un análisis estadístico de los datos de la simulación y los datos experimentales importados desde el módulo LVDAM-ANT. La regresión se realiza tomando los valores del diagrama de radiación simulados con los datos del diagrama de radiación del módulo del laboratorio. La Tabla 1 presenta el resultado del análisis de regresión para los valores de simulador GUI. Según el análisis estadístico, considerando un coeficiente de correlación múltiple de 0,98 es conveniente implementar el simulador para análisis del diagrama de radiación de arreglos lineal de parches rectangulares en antenas microstrip, dado que proporciona resultados muy cercanos a los resultados reales. La interpretación de la Tabla 1, afirma los resultados aproximados de esta herramienta computacional como la simulación de diagramas de radiación

13 ${ }^{\text {th }}$ LACCEI Annual International Conference: "Engineering Education Facing the Grand Challenges, What Are We Doing?" July 29-31, 2015, Santo Domingo, Dominican Republic 
de arreglos parches rectangulares, en cuanto a su uso y aplicación.

TABLA I

ESTADÍSTICA DE LA REGRESIÓN

\begin{tabular}{ll}
\hline \multicolumn{1}{c}{ VARIABLE ESTADísTICA } & \multicolumn{1}{c}{ VALOR } \\
\hline Coeficiente de Correlación Múltiple & $\mathbf{0 , 9 8 2 9 4 1 2 3 1}$ \\
Coeficiente de Determinación $\mathrm{R}^{2}$ & $\mathbf{0 , 9 8 1 2 8 0 3 5 1}$ \\
$\mathrm{R}^{2}$ Ajustado & $\mathbf{0 , 9 8 1 2 4 0 1 3 7}$ \\
Error Típico & $\mathbf{0 , 0 0 2 7 3 0 1 4 8}$ \\
$\mathrm{N}^{\circ}$ de Observaciones & $\mathbf{4 5}$ \\
\hline
\end{tabular}

\section{CONCLUSIONES}

Este artículo presenta el desarrollo de un simulador computacional para estudio del diagrama de radiación de antenas microstrip, arreglo lineal de parche rectangulares. En este contexto, se presentaron las principales características y utilidades del simulador cuyos resultados fueron comparados con datos experimentales.

Se modeló la corriente en el parche microstrip aplicando el método de los momentos (MoM) y se obtuvieron excelentes resultados al ser un modelo que estudia las ondas radiadas (campo eléctrico) y sus características.

Se pudo comprobar que al incrementar el número de parches en una antena, la directividad va ser mayor, además se analizó la influencia de las dimensiones del parche en la directividad.

Es importante resaltar la ventaja que tiene el método de los momentos con respecto a los métodos aproximados modelo de cavidad y línea de transmisión, dado que los modelos aproximados suponen que el campo es nulo por fuera del parche cuando evidentemente no lo es. Sin embargo, esos modelos aproximados permiten calcular con precisión razonable algunos parámetros de la antena.

Se puede determinar que la manera más eficaz de crear antenas microstrip de alta ganancia y mayor directividad, es por medio de arreglos. Permiten al diseñador jugar con el patrón de radiación y crear características únicas en la antena.

\section{REFERENCES}

[1] H. Edward, P. Newman, and P. Tulyathan, "Analysis of Microstrip Antennas Using Moment Methods," IEEE Transactions on Antennas And Propagation, Vol. AP-29, No. 1, January 1981.

[2] A.B. Mutiara, R. Refianti, and Rachmansyah, "Design of Microstrip Antenna for Wireless Communication AT 2.4 Ghz," Journal of Theoretical and Applied Information Technology, Vol. 33, No 2, November 2011.

[3] K. Moussakhani and A. Ghorbani, "A Modified Broadband Transmission Line Model for Rectangular Patch Antennas," International Conference: Microwave and Millimeter Wave Technology, vol. 3, pp. 1041-1043, 2124 April 2008.

[4] N. M. Natashah, et al., "Design of a Microstrip Patch Antenna Using Low Temperature Co-Fired Ceramic Technology," IEEE International Conferenceon Electronic Design, pp. 1 - 3, Dec. 2008.

[5] K. R. Jha and G. Singh, "Analysis of Dielectric Permittivity and Losses of Two- layer Substrate Materials for Microstrip Antenna at $\mathrm{THz}$ Frequency," IEEE Conference Publications, pp. 672 - 675, 2009.
[6] V. S. Kushwah and G. S. Tomar, "Design of Microstrip Patch Antennas Using Neural Network," IEEE Third Asia International Conference on Modelling \& Simulation, pp. 720 - 724, 25 - 29 may. 2009.

[7] K. R. Kashwan, et al., "Design and Characterization of Pin Fed Microstrip Patch Antenna," IEEE Eighth International Conference on Fuzzy Systems and Knowledge Discovery (FSKD), vol. 4, pp. 2258 - 2262, 26 - 28 July 2011.

[8] V. S. Kushwah and G. S. Tomar, "Size reduction of Microstrip Patch Antenna using Defected Microstrip Structures," IEEE International Conference on Communication Systems and Network Technologie, pp. 203 - 207, 3 - 5 June 2011.

[9] V. Bernard and J. P. Izuchukwu, "Microstrip Antenna Design Using Transmission Line Model," International Journal of Emerging Technology and Advanced Engineering, ISSN 2250-2459, ISO 9001:2008 Certified Journal, Vol. 3, Issue 11, Nov. 2013.

[10] P. Singhal and K. Jaimini, "Rectangular Microstrip Patch Antenna Design At $3 \mathrm{GHz}$ Using Probe Feed," International Journal of Emerging Technology and Advanced Engineering, ISSN 2250-2459, ISO 9001:2008 Certified Journal, Vol. 3, Issue 11, Nov. 2013

[11]S. M. Campo, et al., "Simulation and Analysis of the Radiation Pattern of Microstrip Patch-Type Antenna," Latin America Transactions, IEEE (Revista IEEE America Latina), vol. 11, No 1, pp. 341-346, Feb. 2013.

[12]Constantine A. Balanis., Antenna Theory, Analysis and Design, Tercera Edition., John wiley y Sons., 2005.

[13]LVDAM-ANT® - Software, "Lab-Volt - Antenna Training and Measuring System (8092)," Available: http://www.labvolt.com/products/telecommunications/antennas/antennatr aining-and-measuring-system-8092.

13 $^{\text {th }}$ LACCEI Annual International Conference: “Engineering Education Facing the Grand Challenges, What Are We Doing?" July 29-31, 2015, Santo Domingo, Dominican Republic 